
\section{One-sided evolution or two? A reply to Ennos}

\section{SA Hodges and JB Whittall}

Heredity (2008) 100, 541-542; doi:10.1038/hdy.2008.12; published online 20 February 2008

$\mathrm{T}$ hough the term 'coevolution' was coined less than 50 years ago (Ehrlich and Raven, 1964), over 100 years prior to that Darwin first described the process to account for the matching of tongue and tube lengths of bee species and the clover species they visited (Darwin, 1859). He expounded on this process when describing the exceptionally long spur of Angraecum sesquipedale and predicted a moth pollinator with an equally long tongue (Darwin, 1862). Darwin envisioned a 'race' in which long-tongued individuals had a fitness advantage in gaining food from exceptionally longspurred flowers and long-spurred flowers had a fitness advantage in reproduction due to increased pollination. Without a countervailing force, the tongue length of the pollinator and the spur length of the plant would increase due to the reciprocating fitness advantages. When traits of two interacting species such as tongue and floral tube length match tightly, it is easy to assume that Darwin's race, that is, coevolution, is responsible.

We recently tested predictions of Darwin's coevolutionary race model and an alternative, the 'pollinator shift' model (Wasserthal, 1997), as applied to the evolutionary history of spurs among species in the North American Aquilegia clade (Whittall and Hodges, 2007). We concluded that the pollinator shift model is a better fit for the majority of spurlength evolution, that is, species of Aquilegia have primarily evolved to fit the already established tongue lengths of their pollinators. Ennos (2008) provides an excellent summary of our results, and then offers an alternative hypothesis to account for them that combines aspects of both Darwin's coevolutionary race and the pollinator shift hypotheses. He envisions that pollinator shifts do occur but that the process begins when a new pollinator has a similar tongue length as the original pollinator, and thus both are effective pollinators. Then, Darwin's race causes the evolution of longer tongues in the new pollinator class but the original pollinator does not evolve because of some countervailing evolutionary force (for example, body size constraints). Darwin's race then ensues between the plant and the new pollinator until another counterbalancing force prevents the lengthening of the new pollinator's tongue. This counterbalancing force then causes stasis of tongue and spur-length evolution until yet another pollinator class appears with a similar tongue length to carry on Darwin's coevolutionary race.

Ennos' model predicts pollinator shifts followed by stasis in spur length as does the pollinator shift model, which envisions that plants adapt to fit the pre-established tongue length of a pollinator with no reciprocal evolution by the pollinator. Ennos (2008) goes on to claim that the pollinator shift hypothesis 'requires a rather implausible ecological scenario' because it requires that the original pollinator must be absent from the population for many generations. We disagree. Instead, the pollinator shift model only requires that in some part of the plant species' range a longer tongued pollinator becomes the predominant visitor and the cause of most plant reproduction (Whittall and Hodges, 2007). First, bees and hummingbirds do not have to have similar tongue lengths to be similarly effective at pollen transfer of 'bee-adapted' flowers (Castellanos et al., 2003) as Ennos claims. Thus changes in the abundance of these pollinator classes could substantially change the selection regime for floral morphology (Castellanos et al., 2003). Furthermore, temporal and spatial variation in pollinator abundances have been found to be substantial and is thus certainly not implausible (for example, Herrera, 1996; Herlihy and Eckert, 2005; Price et al., 2005; Brunet and Sweet, 2006). Changes in pollinator abundance may occur over long time periods owing to a number of factors, including changes in pollinator migration patterns, extinction of pollinators, changes in community composition or adaptation of plants to a new habitat (see Thomson and Wilson, 2008).
Though such situations may be rare, this does not make them unimportant to plant evolution.

In addition to ecological changes, floral characters can affect the frequency of pollinator visitation. In Aquilegia, hawkmoths do not discriminate between control flowers and those with artificially shortened spurs, but variation in flower orientation and color have very large effects on visitation (Fulton and Hodges, 1999; Hodges et al., 2002). Similarly in two species of Mimulus, introgression of alternate quantitative trait locus alleles (QTL) for flower color caused dramatic changes in the frequency of visitation by bees and hummingbirds (Bradshaw and Schemske, 2003). Thus, changes in traits other than nectar spurs may precipitate shifts in pollinator visitation rates. Such changes would be especially likely to cause shifts when the new pollinator was already particularly abundant (Bradshaw and Schemske, 2003). Once the setting for more frequent visitation is created then there would be very strong selection for other floral traits, such as nectar spur length, to become optimized for plant reproduction via the new pollinator (Thomson and Wilson, 2008).

There are further aspects of Darwin's coevolutionary race model, alone or as part of Ennos's hybrid model, which seem to us to make it an unlikely explanation for the majority of spurlength evolution in the North American Aquilegia clade (or more generally, floral tube-length evolution). For example, pollinators such as hummingbirds likely already had tongue lengths similar to those found today prior to their first association with bee-adapted Aquilegia. One clade of hummingbirds, called beehummingbirds, is comprised of two sister clades, one including all North American species and the other including primarily South American species (McGuire et al., 2007). Phylogenetic analysis indicates that the ancestor of bee-hummingbirds occurred in South or Central America (McGuire et al., 2007). This ancestor also likely had a bill morphology similar to extant bee-hummingbird species because species in both sister clades have similar bill morphology (around 1.5 to less than $2.0 \mathrm{~cm}$; Colwell, 2000). Thus, bill size of hummingbirds was likely established prior to their invasion of North America and an interaction with Aquilegia (or other North American species that became adapted to hummingbirds). Furthermore, molecular dating indicates that bee-hummingbirds originated 
about 6 mya (Bleiweiss, 1998), while the origin of hummingbird pollination in Aquilegia was likely to have been far more recent at around 1.7 mya or less (Hodges et al., 2004; Kay et al., 2006). Taken together, these results strongly suggest that bill morphology was established prior to an association with Aquilegia and that it did not substantially change thereafter. We have already made a similar argument concerning hawkmoth tongue lengths (Whittall and Hodges, 2007).

Another reason we doubt coevolutionary hypotheses provide a major explanation for most spur-length evolution is because the interaction between plant and pollinator is likely to be very asymmetrical. Hummingbirds and hawkmoths visit a large number of species (for example, Grant, 1983, 1994) and thus they do not have a tight dependency on any one plant species. For example, the longest spurred Aquilegia species, A. longissima, belongs to a guild of unrelated species from six distinct genera in the desert (southwest USA), all with floral tubes greater than $9 \mathrm{~cm}$, which by and large share the same hawkmoth pollinators (Grant, 1983; Grant and Grant, 1983). Thus, the ability of the longest tongued individuals to reach a bit more nectar from the longest spurred individuals of a single species would provide a rather small fitness advantage for the pollinator, at best. In contrast, reproduction in most plants is dependent on the visitation of pollinators and thus selection will be quite strong to optimize floral morphology to maximize pollen transfer. Thus, unrelated plant species visited by the same pollinator will likely converge on a similar floral morphology, such as tube or spur length. Similar arguments have been made during a previous discussion of Darwin's coevolutionary race and the pollinator shift hypothesis (Jermy, 1999). Finally, Ennos' model requires a new species of hummingbird or hawkmoth for each transition to a new pollinator so that Darwin's race can be run each time anew. Given the large number of shifts from bee to hummingbird and from hummingbird to hawkmoth pollinated plant species in North America (for example, Grant, 1994; Whittall and Hodges, 2007; Thomson and Wilson, 2008) and the much smaller number of hummingbird and hawkmoth species, this seems unlikely as a general explanation for the evolution of tube and tongue lengths.
While we believe that the pollinator shift hypothesis accounts for the majority of spur-length evolution in Aquilegia, and likely many other species as well, some spur and tongue lengths may well be due to Darwin's coevolutionary race. How would one identify such instances? We agree with Ennos that complementary, and more specifically, phylogenetic studies of plants and pollinators will be needed. If evolution among species or populations of both plants and pollinators indicates increasing lengths during the same time period and in the same geographical area, then this would provide strong evidence for Darwin's race. Even still, it will be necessary to establish that the respective fitness advantages are due to the plantpollinator interaction rather than some other factor (for example, Wasserthal, 1997; Borrell, 2005; Strauss and Whittall, 2006).

Professor SA Hodges is at the Department of Ecology, Evolution and Marine Biology, University of California, Santa Barbara, CA, USA and JB Whittall is at the Department of Biology, Santa Clara University, Santa Clara, CA, USA

\section{e-mail: hodges@lifesci.ucsb.edu}

Bleiweiss R (1998). Tempo and mode of hummingbird evolution. Biol J Linn Soc 65: 63-76.

Borrell BJ (2005). Long tongues and loose niches: evolution of Euglossine bees and their nectar flowers. Biotropica 37: 664-669.

Bradshaw HD, Schemske DW (2003). Allele substitution at a flower colour locus produces a pollinator shift in monkeyflowers. Nature 426: 176-178.

Brunet J, Sweet HR (2006). Impact of insect pollinator group and floral display size on outcrossing rate. Evolution 60: 234-246.

Castellanos MC, Wilson P, Thomson JD (2003). Pollen transfer by hummingbirds and bumblebees, and the divergence of pollination modes in Penstemon. Evolution 57: 2742-2752.

Colwell RK (2000). Rensch's rule crosses the line: convergent allometry of sexual size dimorphism in hummingbirds and flower mites. Am Nat 156: 495-510.

Darwin C (1859). On the Origin of Species by Means of Natural Selection, or the Preservation of Favoured Races in the Struggle for Life Facsimile of the 1st edn. Harvard University Press: Cambridge.

Darwin C (1862). The Various Contrivances by which Orchids are Fertilized by Insects. John Murray: London.

Ehrlich PR, Raven PH (1964). Butterflies and plants: a study in coevolution. Evolution 18: 586-608.

Ennos RA (2008). Spurred on by pollinators. Heredity 100: 3-4.

Fulton M, Hodges SA (1999). Floral isolation between Aquilegia formosa and A. pubescens. Proc R Soc Lond B 266: 2247-2252.

Grant V (1983). The systematic and geographic distribution of hawkmoth flowers in the temperate North American flora. Bot Gaz 144: $439-449$.
Grant V (1994). Historical development of ornithophily in the western North American flora. Proc Natl Acad Sci USA 91: 10407-10411.

Grant V, Grant KA (1983). Hawkmoth pollination of Mirabilis longiflora (Nyctaginaceae). Proc Natl Acad Sci USA 80: 1298-1299.

Herlihy CR, Eckert CG (2005). Evolution of selffertilization at geographic range margins? A comparison of demographic, floral, and mating system variables in central vs peripheral populations of Aquilegia canadensis (Ranunculaceae). Am J Bot 92: 744-751.

Herrera CM (1996). Floral traits and plant adaptation to insect pollinators: a devil's advocate approach. In: Lloyd DG, Barrett SCH (eds.). Floral Biology: Studies on Floral Evolution in Animal-Pollinated Plants. Chapman and Hall: New York. pp 65-87.

Hodges SA, Whittall JB, Fulton M, Yang JY (2002). Genetics of floral traits influencing reproductive isolation between Aquilegia formosa and A. pubescens. Am Nat 159: S51-S60.

Hodges SA, Fulton M, Yang JY, Whittall JB (2004). Verne Grant and evolutionary studies of Aquilegia. New Phyt 161: 113-120.

Jermy T (1999). Deep flowers for long tongues: a final word. Trends Ecol Evol 14: 34.

Kay KK, Whittall JB, Hodges SA (2006). A survey of nuclear ribosomal internal transcribed spacer substitution rates across angiosperms: an approximate molecular clock with life history effects. BMC Evol Biol 6: 36.

McGuire JA, Witt CC, Altshuler DL, Remsen Jr JV (2007). Phylogenetic systematics and biogeography of hummingbirds: Bayesian and maximum likelihood analyses of partitioned data and selection of an appropriate partitioning strategy. Syst Biol 56: 837-856.

Price MV, Waser NM, Irwin RE, Campbell DR, Brody AK (2005). Temporal and spatial variation in pollination of a montane herb: a seven-year study. Ecology 86: 2106-2116.

Strauss SY, Whittall JB (2006). Non-pollinator agents of selection on floral traits. In: Harder L, Barrett SCH (eds). Ecology and Evolution of Flowers. Oxford University Press: Oxford, pp 120-138.

Thomson JD, Wilson P (2008). Explaining evolutionary shifts between bee and hummingbird pollination: convergence, divergence, and directionality. Int J Plant Sci 169: 23-38.

Wasserthal LT (1997). The pollinators of the Malagasy star orchids Angraecum sesquipedale, $A$. sororium and $A$. compactum and the evolution of extremely long spurs by pollinator shift. Bot Acta 110: 343-359.

Whittall JB, Hodges SA (2007). Pollination shifts drive increasingly long nectar spurs in columbine flowers. Nature 447: 706-709.

\section{Editor's suggested reading}

Bangert RK, Lonsdorf EV, Wimp GM, Shuster SM, Fischer D, Schweitzer JA et al. (2008). Genetic structure of a foundation species: scaling community phenotypes from the individual to the region. Heredity 100: 121-131.

Dubuffet A, Dupas S, Frey F, Drezen J-M, Poirié M, Carton Y et al. (2007). Genetic interactions between the parasitoid wasp Leptopilina boulardi and its Drosophila hosts. Heredity 98: 21-27.

Gomulkiewicz R, Drown DM, Dybdahl MF, Godsoe W, Nuismer SL, Pepin KM et al. (2007). Dos and don'ts of testing the geographic mosaic theory of coevolution. Heredity 98: 249-258.

Montoya JM (2007). Evolutionary studies: evolution within food webs: the possible and the actual. Heredity 99: 477-478. 